\title{
Techniques de frustration de fluorescence dans le VUV : Effet du couplage spin-orbite dans l'excimère de krypton
}

\author{
M. Hemici, R. Saoudi, E. Descroix, E. Audouard et P. Laporte \\ Laboratoire Traitement du Signal et Instrumentation, URA 842 du CNRS, 23 rue du Dr Paul \\ Michelon, 42023 Saint-Etienne cedex 2, France
}

\begin{abstract}
Résumé: La frustration de la fluorescence VUV de l'excimère de krypton permet de sonder les états moléculaires de $\mathrm{Kr}_{2}{ }^{*}$ dans le domaine de longueur d'onde $955-995 \mathrm{~nm}$. Le calcul ab initio d'un spectre synthétique a permis l'attribution des bandes observées sans ambiguité. Ces résultats permettent de mettre en évidence l'effet du couplage spin-orbite dans les gaz rares lourds.
\end{abstract}

\begin{abstract}
The Laser Reduced VUV fluorescence of krypton excimer allows to probe molecular states in the infrared wavelength range. A clear assignment is obtained by using a comparison of the five observed bands with $a b$ initio calculated spectra. The effect of the spin orbit coupling in heavy rare gas is thus evidenced
\end{abstract}

Dans les dernières années, un large effort à la fois théorique et experimental a permis un progrès déterminant dans la compréhension des structures moléculaires des excimères de gaz rares et particulièrement de $\mathrm{Kr}_{2}{ }^{*} 1,2$. Dans ce cadre général, nous présentons une contribution expérimentale basée sur la mise en oeuvre de techniques particulièrement adaptées à l'étude de ces molécules, qu'il n'est pas possible d'étudier directement depuis l'état de base répulsif à courte distance internucléaire, ainsi qu'une analyse théorique fondée sur les résultats du calcul $a b$ initio des potentiels moléculaires ${ }^{3}$.

La nature prédissociée des états sondés à courte distance internucleaire et les absorptions atomiques résiduelles rendent délicate l'obtention de spectre d'absorption moléculaire. La mise en oeuvre de techniques de détection de la frustration de la fluorescence VUV du premier état de l'excimère ${ }^{3} \sum_{u^{+}}+\left(1_{u} 0_{u^{-}}\right)$a permis d'obtenir de tels spectres. Dans ce travail, nous étudions les transitions moléculaires entre ${ }^{3} \Sigma_{\mathbf{u}^{+}}{ }^{(}\left(1_{\mathbf{u}} 0_{\mathbf{u}^{-}}\right)$vers les premiers états de symétrie $\mathrm{g}\left({ }^{3}, 1 \Pi_{\mathrm{g}}\right)$ associés à des puits de potentiels semblables au puits de l'état de départ. Après couplage spin-orbite, ces états se décomposent en cinq états différents notés $0 \mathrm{~g}^{+}, 0 \mathrm{~g}^{-}, 2 \mathrm{~g}$ et deux notés $1 \mathrm{~g}$. Les transitions lié-lié correspondantes se situent dans le domaine de l'infrarouge (autour de $970 \mathrm{~nm}$ ). Le dispositif expérimental permet d'obtenir une résolution de $0.2 \mathrm{~cm}^{-1}$ dans un domaine de pression allant de 150 a $2000 \mathrm{hPa}$. Un exemple de spectre obtenu est présenté sur la figure 1. Le spectre théorique est obtenu par un calcul des facteurs de Franck-Condon associés aux résultats du calcul $a b$ initio des moments de transition, et la prise en compte, d'une façon semi-empirique, d'une largeur de prédissociation. La confrontation théorie expérience illustre la bonne précision du calcul ab initio et 
précision du calcul $a b$ initio et permet l'attribution non ambiguë des cinq raies observées à des transitions depuis $3 \sum_{\mathrm{u}}+\left(1_{\mathrm{u}} 0_{\mathrm{u}}{ }^{-}\right)$vers les cinq états $\mathrm{g}$ attendus. Une analyse à plus haute résolution montre que la transition dont l'énergie est la plus faible correspond à un état peu prédissocié de la molécule. Une analyse en terme de transitions vibroniques est alors possible, un tel travail est en cours.

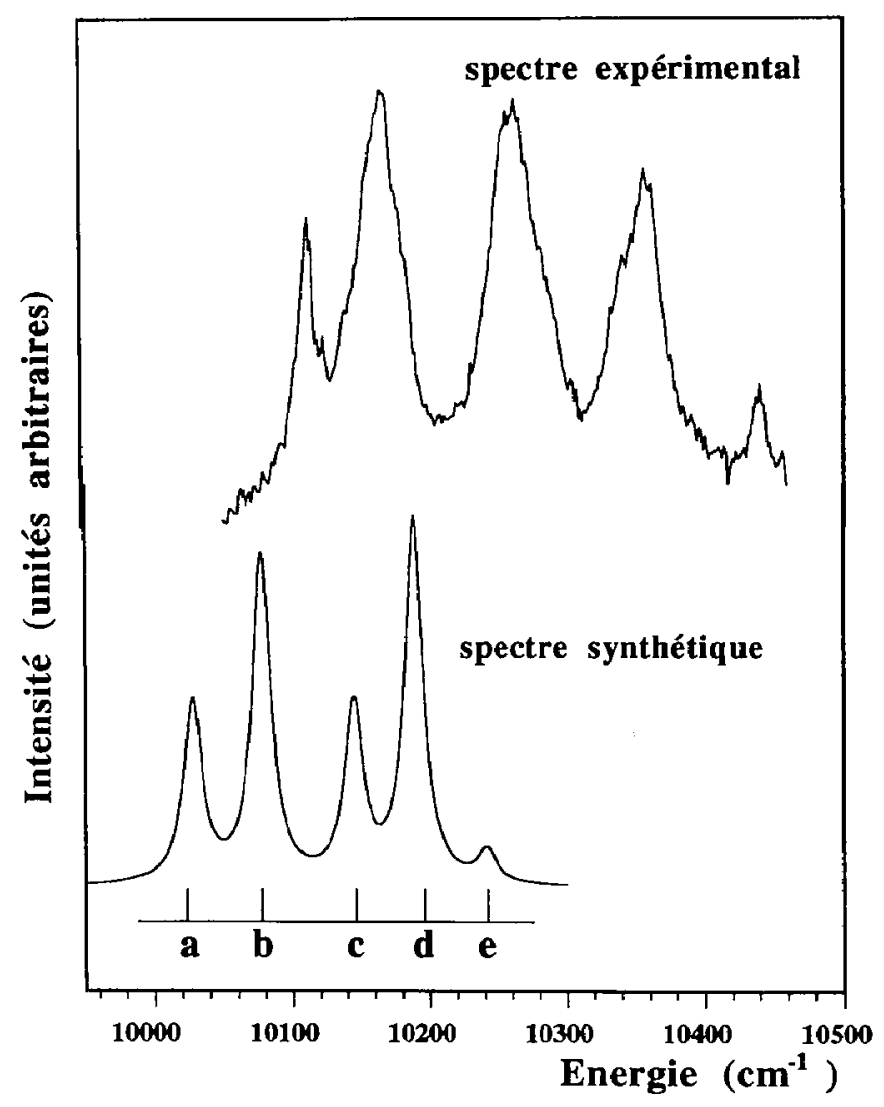

Figure 1: Comparaison des spectres expérimental et synthétique autour de $970 \mathrm{~nm}$. La pression est de $400 \mathrm{hPa}$ et la résolution spectrale est de $0.7 \mathrm{~cm}^{-1}$. Les lettres a, b, c, $\mathrm{d}$, e repèrent les transitions depuis l'état ${ }^{3} \sum_{\mathrm{u}^{+}}+\left(1_{\mathrm{u}} \mathrm{O}_{\mathrm{u}}{ }^{-}\right)$vers les états (III) $\mathrm{g}_{\mathrm{g}}{ }^{-},(\mathrm{IV}) 1_{\mathrm{g}}$, (IV) $0 \mathrm{~g}^{+}$, (II) $2_{\mathrm{g}}$, (V) $1 \mathrm{~g}$ (notations de la Ref. 3 )

\section{REFERENCES}

[1] D.J. Kane, C.C. Abele, R.D. Fraser, D.C. Shannon, J.G. Eden and M.L. Ginter, J. Chem. Phys. 99, (1993), 99-112.

[2] M. Hemici, R. Saoudi, E. Descroix, E. Audouard, and P. Laporte,

J Phys IV, France, 4, (1994), C4, 755-758.

[3] E. Audouard and F. Spiegelmann,

J. Chem. Phys. 94, (1991), 6102-6124. 